\title{
Endoscopic Creation of a Neochimneycystotomy: A Case Report of a Ureteroileal Chimney Stricture
}

\author{
Christine W. Liaw, MD, Jacob Bamberger, BS, Kasmira Gupta, and Mantu Gupta, MD
}

\begin{abstract}
Background: There are many nonmalignant complications after urinary reconstruction. Anastomotic strictures and redundancy of an ileal chimney are such. A patient with both issues might necessitate an open surgical approach; yet endoscopic techniques are more attractive for these older frail patients.

Case Presentation: A 61-year-old woman with a history of bladder cancer who underwent radical cystectomy and neobladder creation now develops left hydronephrosis and a redundant ileal chimney with severe metabolic acidosis. She underwent endoscopic creation of a neochimneycystotomy.

Conclusion: The refinement of endoscopic techniques moves the field of surgery away from open surgery, which is beneficial for patients. This endoscopic technique treated the anastomotic stricture as well as redundant ileal chimney in a novel way that has not been reported previously in the literature.
\end{abstract}

Keywords: ileal chimney neobladder, anastomotic stricture, endoscopic treatment

\section{Introduction}

$\mathbf{R}$ ADICAL CYSTECTOMY WITH urinary diversion can be curative for bladder cancer, but is accompanied by a host of complications. Redundancy of the bowel segment (such as the ileal chimney for a neobladder) and anastomotic stricture are common complications. Techniques for treatment of these complications fall into two general categories: endoscopic and open. More conservative techniques are attractive for a certain population who, having undergone treatment for bladder cancer, may be older and more frail. A patient who develops both complications may be relegated to an open surgical repair. However, we present a minimally invasive endoscopic technique.

\section{Presentation of Case}

A 61-year-old woman with a history of urothelial carcinoma (diagnosed with transurethral resection of bladder tumor resulting in pathology $\mathrm{T} 2$, negative metastatic workup on imaging, underwent neoadjuvant chemotherapy with ddMVAC $\times 4$ cycles, underwent radical cystectomy and neobladder creation, complicated by neobladdervaginal fistula then underwent repair) was found to have left hydronephrosis with a tortuous and redundant ileal chimney on surveillance imaging. Imaging from before the radical cystectomy, performed for oncologic staging, revealed no hydronephrosis. Renal scan showed left obstruction (split function of left kidney was $17 \%$ with diuretic excretion $T 1 / 2$ 36.5 minutes). She also had poor drainage of the ileal chimney because of kinking and redundant length. Creatinine peaked at 1.21 (baseline 0.7 ). She also had metabolic derangements (acidosis, decreased bicarbonate, and elevated chloride), thought to originate from urinary stasis in the redundant ileal chimney. She underwent interventional radiology percutaneous nephroureteral tube placement, which was later internalized to a Double-J ureteral stent. This was managed in the interim with a chronic ureteral stent and routine exchanges. However, because of the extensive length of the ileal chimney, the ureteral stents were never long enough to traverse from the kidney all the way into the neobladder.

She was referred for endoscopic minimally invasive reconstruction and repair at this time. She underwent endoscopic left ureteroneocystostomy with ureteroplasty. Through cystoscopy into the neobladder, the neochimneycystotomy was created by incising the right lateral aspect of the neobladder utilizing fluoroscopy and the existing stent as a guiding target. Contrast confirmed entrance into the ileal chimney (Fig. 1A). Now the majority of the redundant ileal chimney was bypassed. The existing ureteral stent was severely encrusted and required extensive cystolitholapaxy and antegrade percutaneous nephrolithotripsy. The left ureteral-ileal chimney anastomotic stricture was noted again on ureteroscopy, and ureteroplasty with balloon dilation was performed (Fig. 1B). A nephrostomy tube, tandem ureteral

Department of Urology, Icahn School of Medicine at Mount Sinai, New York, New York, USA. 
FIG. 1. (A) Balloon dilation of the neochimney neobladder connection. (B) Balloon dilation of the ureteral-ileal chimney anastomotic stricture.
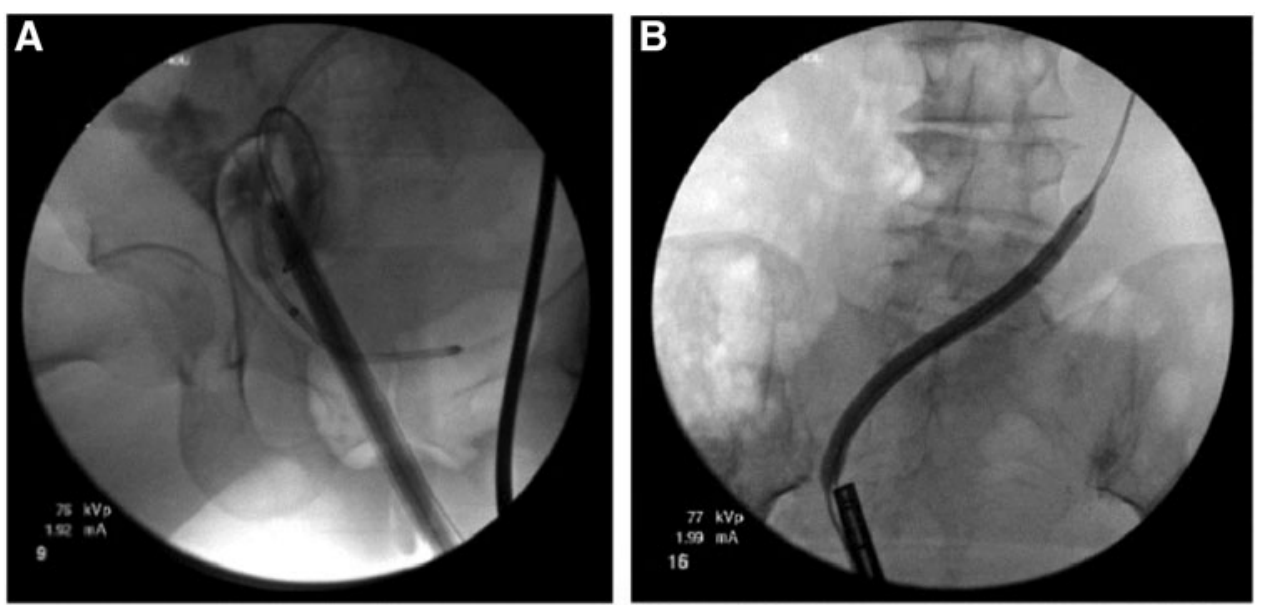

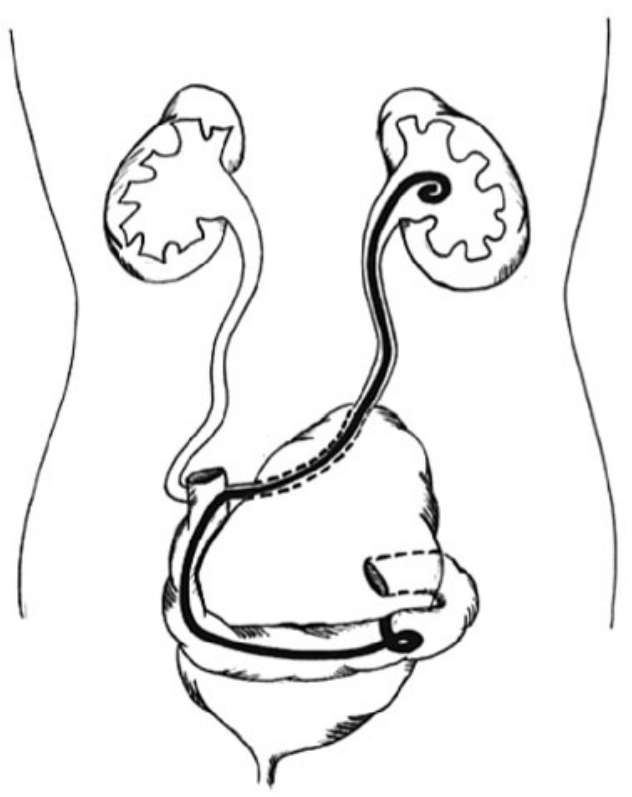

FIG. 2. Illustration of neochimney C. stents, and Foley catheter were left at the end of the procedure. Figure 2 is an illustration of the neochimneycystotomy.

The Foley was removed on postoperative day 3. Office cystoscopy 1 month later revealed that the neochimneycystotomy was open but tight around the ureteral stents. A guidewire was able to be passed next to the stents into chimney but unable to go up to left kidney despite manipulation.

The decision was made to undergo cystoscopy with incision of the stricture. The stricture was cannulated with a guidewire and Pollack catheter, which were then manipulated into the left collecting system. The tight neochimneycystotomy was incised with a Collins knife. The pre-existing stents were removed and exchanged for a single Double-J stent. A Foley catheter was placed going through the ureteroplasty site with the balloon being inflated in the ileal chimney; side holes were cut proximal to the balloon for simultaneous drainage of the neobladder (Fig. 3).

Repeat office cystoscopy was performed 3 months after the initial procedure. The flexible cystoscope was able to pass alongside the stent up to the left ureteral-ileal chimney anastomosis. The neochimneycystotomy was widely patent around the ureteral stent. The stent was removed. Repeat CT scan revealed resolution of the left hydroureter and marked improvement of hydronephrosis. The CT scan also shows contrast draining from the ileal chimney into the neobladder.
FIG. 3. (A) Pre-existing stents in place with wire going up into left collecting system. (B) End of procedure with new stent and Foley catheter draining ileal chimney and neobladder.
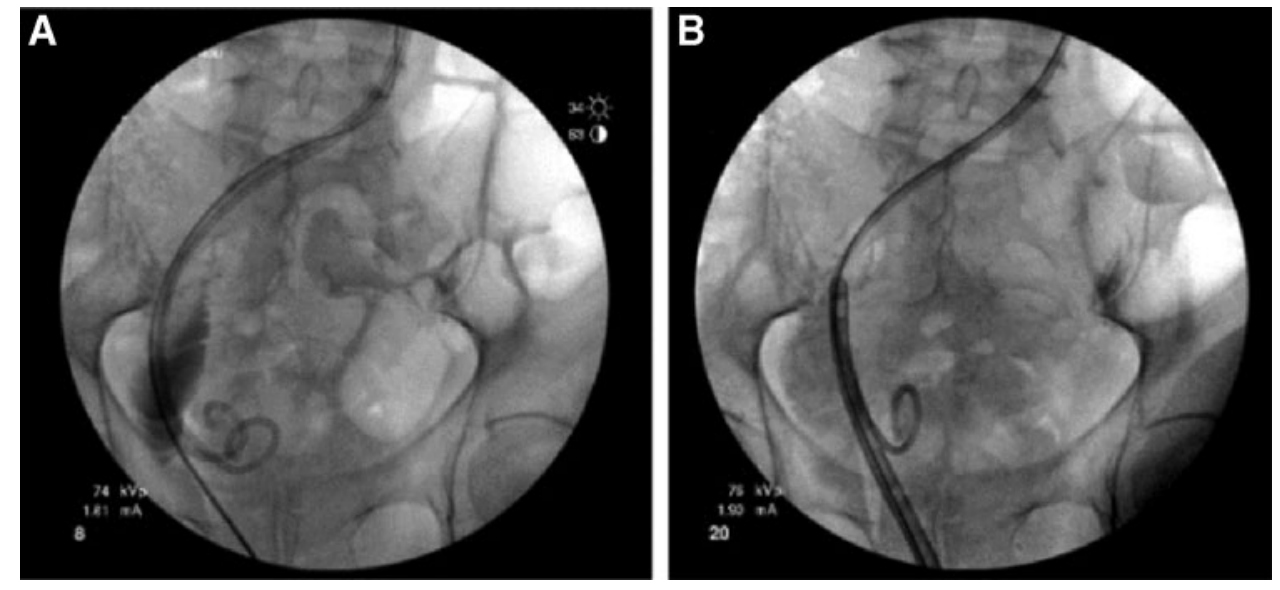


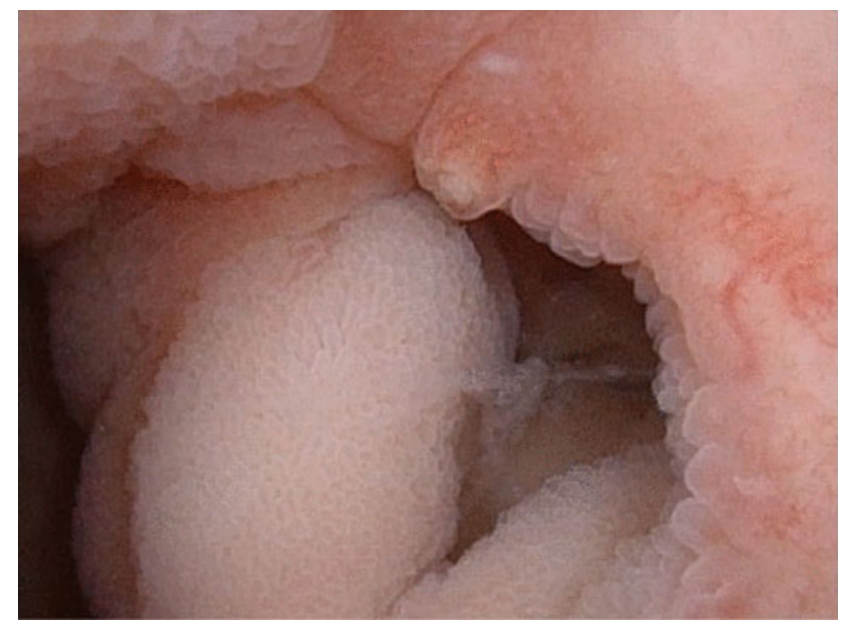

FIG. 4. Patent neochimney neobladder.

Latest repeat office cystoscopy 6 months after the initial procedure revealed continued patency of the neochimneycystotomy (Fig. 4).

\section{Discussion}

Nonmalignant anastomotic strictures after urinary diversion are a common complication, incidence $5 \%-10 \% .{ }^{1}$ These patients can present with flank pain, infection, or a rise in creatinine. Most patients initially undergo endoscopic intervention (balloon dilation and laser incision), with a $26 \%-$ $51.3 \%$ success rate. $^{2}$ Open surgical repair (stricture resection and reanastomosis, ureteral reimplant) is obviously more invasive but with a much higher success rate at $76 \%-90 \%$. $^{2,3}$ One centimeter stricture length is the cutoff for effective endoscopic intervention, with a precipitous drop in success rate with longer strictures. ${ }^{2,3}$

The aforementioned techniques are the traditional reparative procedures for managing most strictures. Longer strictures require some sort of interposition. Recently, Rayn et al. reports on the "Reverse 7." 4 Their patient had a radical cystectomy with neobladder creation as well. The patient developed bilateral anastomotic strictures. An ileal segment was fashioned in the shape of a mirror image 7. The top bar of the seven anastomosed to each renal pelvis and the bottom portion anastomosed to the top of the neobladder. Although only immediate postoperative course was reported, the "Reverse 7" represents an innovative technique to treat particularly complicated stricture patients.

Metabolic derangements are well recognized in urinary diversion patients. Use of the ileal creates hyperchloremic metabolic acidosis. The long-term treatment is to decrease urinary stasis and the amount of time the urine is in contact with the bowel segment. Some patients are managed with a catheterization regimen. However, this patient's redundant anatomy prevented catheterization from being a useful treatment. Her metabolic acidosis improved after the procedures, along with her general symptoms of malaise and fatigue.

This neochimneycystotomy has great short-term success a few months postoperatively. The patient stayed in the hospital for one night after first procedure and four nights after the second procedure, but only because of social work reasons unrelated to urologic care. These otherwise could be ambulatory procedures. In addition, the first procedure was not as aggressive with creating the opening between the ileal chimney and neobladder. If the Collins knife was used initially, this may have pre-empted a second procedure.

\section{Conclusion}

The refinement of endoscopic techniques moves the field of surgery away from open surgery, which is beneficial for patients. This endoscopic technique treated the anastomotic stricture as well as redundant ileal chimney in a novel way that has not been reported previously in the literature.

\section{Disclosure Statement}

No competing financial interests exist.

\section{Funding Information}

No funding was received for this article.

\section{References}

1. Studer UE, Burkhard FC, Schumacher M, et al. Twenty years experience with an ileal orthotopic low pressure bladder substitute-Lessons to be learned. J Urol 2006;176:161.

2. Schondorf D, Meierhans-Ruf S, Kiss B, Giannarini G, Thalmann GN, Studer UE, Roth B. Ureteroileal strictures after urinary diversion with an ileal segment-Is there a place for endourological treatment at all? J Urol 2013;190: 585-590.

3. DiMarco DS, LeRoy AJ, Thieling S, Bergstralh EJ, Segura JW. Long-term results of treatment for ureteroenteric strictures. Urology 2001;58:909-913.

4. Rayn KN, Ritchie C, Folio LR, Stamatakis L, Verghese MM, Agarwal PK. Bilateral ureteroenteric strictures: A case of the "Reverse 7." Urology 2018;118:e3-e4.

$$
\begin{array}{r}
\text { Address correspondence to: } \\
\text { Christine W. Liaw, MD } \\
\text { Department of Urology } \\
\text { Icahn School of Medicine at Mount Sinai } \\
\text { One Gustave L. Levy Place } \\
\text { New York, NY 10029 } \\
\text { USA }
\end{array}
$$

E-mail: christine.liaw@mountsinai.org

Abbreviation Used
$\mathrm{CT}=$ computed tomography

Cite this article as: Liaw CW, Bamberger J, Gupta K, Gupta M (2020) Endoscopic creation of a neochimneycystotomy: A case report of a ureteroileal chimney stricture, Journal of Endourology Case Reports 6:3, 163-165, DOI: 10.1089/cren.2019.0173 